\title{
Artificial Intelligence for Computer Games
}

\author{
Abdennour El Rhalibi, ${ }^{1}$ Kok Wai Wong, ${ }^{2}$ and Marc Price ${ }^{3}$ \\ ${ }^{1}$ School of Computing and Mathematical Sciences, Liverpool John Moores University, Byrom Street, Liverpool L3 3AF, UK \\ ${ }^{2}$ School of Information Technology, Murdoch University, 90 South Street, Murdoch WA 6150, Australia \\ ${ }^{3}$ BBC Research, Kingswood Warren, Tadworth, Surrey KT20 6NP, UK \\ Correspondence should be addressed to Abdennour El Rhalibi, a.elrhalibi@ljmu.ac.uk
}

Received 15 December 2008; Accepted 15 December 2008

Copyright (C) 2009 Abdennour El Rhalibi et al. This is an open access article distributed under the Creative Commons Attribution License, which permits unrestricted use, distribution, and reproduction in any medium, provided the original work is properly cited.

Artificial intelligence (AI) in computer games covers the behaviour and decision-making process of game-playing opponents (also known as nonplayer character or NPC). Current generations of computer and video games offer an amazingly interesting testbed for AI research and new ideas. Such games combine rich and complex environments with expertly developed, stable, physics-based simulation. They are real-time and very dynamic, encouraging fast and intelligent decisions. Computer games are also often multiagents, making teamwork, competition, and NPC modelling key elements to success. In commercial games, such as action games, role-playing games, and strategy games, the behaviour of the NPC is usually implemented as a variation of simple rule-based systems. With a few exceptions, machine-learning techniques are hardly ever applied to stateof-the-art computer games. Machine-learning techniques may enable the NPCs with the capability to improve their performance by learning from mistakes and successes, to automatically adapt to the strengths and weaknesses of a player, or to learn from their opponents by imitating their tactics.

In this special issue, we introduce a number of interesting papers contributing to a wide range of these topics and reflecting the current state of AI for Computer Game in academia. A total of 20 papers have been submitted to this special issue, of which 9 high-quality papers have been accepted after the peer review process.

This special issue starts with the first paper "Performance simulations of moving target search algorithms" by Peter Kok Keong Loh et al. In this paper, the authors focused on the design of moving target search (MTS) algorithms for computer generated bots. MTS algorithms pose important challenges as they have to satisfy rigorous requirements which involve combinatorial computation and performance. In this paper, the authors investigate the performance and behaviour of existing moving target search algorithms when applied to search-and-capture gaming scenarios. As part of the investigation, they also introduce a novel algorithm known as abstraction MTS. They conduct performance simulations with a game bot and moving target within randomly generated mazes of increasing sizes and reveal that abstraction MTS exhibits competitive performance even with large problem spaces.

The second paper is proposed by Julio Clempner and is entitled "A shortest-path lyapunov approach for forward decision processes." In this paper, the author presents a formal framework for shortest-path decision process problem representation. Dynamic systems governed by ordinary difference equations described by Petri nets are considered. The trajectory over the net is calculated forward using a discrete Lyapunov-like function. Natural generalizations of the standard outcomes are proved for the deterministic shortestpath problem. In this context, the authors are changing the traditional cost function by a trajectory-tracking function which is also an optimal cost-to-target function for tracking the net. This makes an important contribution in the conceptualization of the problem domain. The Lyapunov method introduces a new equilibrium and stability concept in decision process for shortest path.

The third paper is entitled "Fractal analysis of stealthy pathfinding aesthetics" authored by Coleman Ron. In this paper, the author uses a fractal model to analyze aesthetic values of a new class of obstacle prone or "stealthy" pathfinding which seeks to avoid detection, exposure, and 
openness in videogames. This study is interesting since in general the artificial intelligence literature has given relatively little attention to aesthetic outcomes in pathfinding. The data reported, according to the fractal model, suggests that stealthy paths are statistically significantly unique in relative aesthetic value when compared to control paths. The author also shows that paths generated with different stealth regimes are also statistically significantly unique. These conclusions are supported by statistical analysis of model results on experimental trials involving pathfinding in randomly generated, multiroom virtual worlds.

The next paper is proposed by Frank Dignum et al. and discusses "Games and agents: designing intelligent Gameplay." Multiagent system research offers a promising technology to implement cognitive intelligent NPC's. However, the technologies used in game engines and multiagent platforms are not readily compatible due to some inherent differences of concerns. Where game engines focus on realtime aspects and thus propagate efficiency and central control, multiagent platforms assume autonomy of the agents. Increased autonomy and intelligence may offer benefits for a more compelling gameplay and may even be necessary for serious games. However, it raises problems when current game design techniques are used to incorporate state of the art multiagent system technology. In this paper, the authors focused on three specific problem areas that arise from this difference of view: synchronization, information representation, and communication. They argue that current attempts for integration still fall short on some of these aspects. They show that to fully integrate intelligent agents in games, one should not only use a technical solution, but also a design methodology such as OperA, that is amenable to agents.

The fifth paper presents "A multiagent potential fields based bot for real-time strategy games" authored by Johan Hagelback et al. The paper discusses bots for real-time strategy (RTS). A bot controls a number of units that will have to navigate in a partially unknown environment, while at the same time avoid each other, search for enemies, and coordinate attacks to fight them down. "Potential fields" is a technique originating from the area of robotics where it is used in controlling the navigation of robots in dynamic environments. The authors present a multiagent potential field based bot architecture which is evaluated in two different real-time strategy game settings and compare it, in terms of performance, and configurability, to other state-ofthe-art solutions. The authors show that the solution is a highly configurable bot which can match the performance of traditional RTS bots. They also show that a multiagent potential field-based bot is highly competitive in a resource gathering scenario.

The next paper is "Combining artificial intelligence methods for learning bots in a real time strategy game" authored by Robin Baumgarten et al. The authors describe an approach to simulate human game-play in strategy games using a variety of AI techniques, including simulated annealing, decision tree learning, and case-based reasoning. They have implemented an AI-bot that uses these techniques to form a novel approach to plan fleet movements and attacks in DEFCON, a nuclear war simulation strategy game released in 2006 by Introversion Software Ltd, Surrey, UK. They describe how the AI-bot operates, and the experimentation they have performed in order to determine an optimal configuration for it. With this configuration, the proposed AI-bot beats Introversion's finite state machine automated player in $76.7 \%$ of 150 matches played.

The seventh paper is "Enhancing artificial intelligence on a real mobile game" by Fabio Aiolli et al. Mobile gaming represents a killer application that is attracting millions of subscribers worldwide; yet, several technical issues in this context remain unsolved. One of the aspects crucial to the commercial success of a game is ensuring an appropriately challenging artificial intelligence (AI) algorithm against which to play. However, creating this component is particularly complex as classic search AI algorithms cannot be employed by limited devices such as mobile phones or, even on more powerful computers, when considering imperfect information games (i.e., games in which participants have not a complete knowledge of the game state at any moment). In this paper, the authors propose to solve the imperfect information game issue by resorting to a machine learning algorithm which uses profiling functionalities in order to infer the missing information, and making the AI able to efficiently adapt its strategies to the human opponent. They studied a very simple and computationally light machine learning method that can be employed with success, enabling $\mathrm{AI}$ improvements for imperfect information games even on mobile phones. They present results on a simple game called Ghosts which show the ability of their algorithm to quickly improve its own predictive performance as the number of games against the same human opponent increases. A mobile phone-based version of the game has been also created which can be played either against another player or against the AI algorithm.

The eighth paper is "Breeding terrains with genetic terrain programming - the evolution of terrain generators" by Miguel Frade et al. Although a number of terrain generation techniques have been proposed during the last few years, all of them have some key constraints. Modelling techniques depend highly upon designer's skills, time, and effort to obtain acceptable results, and cannot be used to automatically generate terrains. The simpler methods allow only a narrow variety of terrain types and offer little control on the outcome terrain. The Genetic Terrain Programming technique, proposed, based on evolutionary design with genetic programming, allows designers to evolve terrains according to their aesthetic intentions or desired features. This technique evolves terrain programmes (TPs) that are capable of generating a family of terrains - different terrains that consistently present the same morphological characteristics. This paper presents a study about the persistence of morphological characteristics of terrains generated with different resolutions by a given TP. Results show that it is possible to use low resolutions during the evolutionary phase without compromising the outcome and that terrain macrofeatures are scale invariant.

Finally, the last paper is "Fine-tuning parameters for emergent environments in games using artificial intelligence" 
authored by Vishnu Kotrajaras et al. This paper presents the design, development, and test results of a tool for adjusting properties of emergent environment maps automatically according to a given scenario. Adjusting properties for a scenario allows a specific scene to take place while still enables players to meddle with emergent maps. The tool uses genetic algorithm and steepest ascent hill-climbing to learn and adjust map properties. The authors shows that using the proposed tool, the need for time consuming and labourintensive parameter adjustments when setting up scenarios in emergent environment maps, is greatly reduced. The tool works by converting the paths of events created by users for a map to the properties of the map that plays out the scenario set by the given paths of events. Test results show good properties preservation.

Abdennour El Rhalibi

Kok Wai Wong

Marc Price 

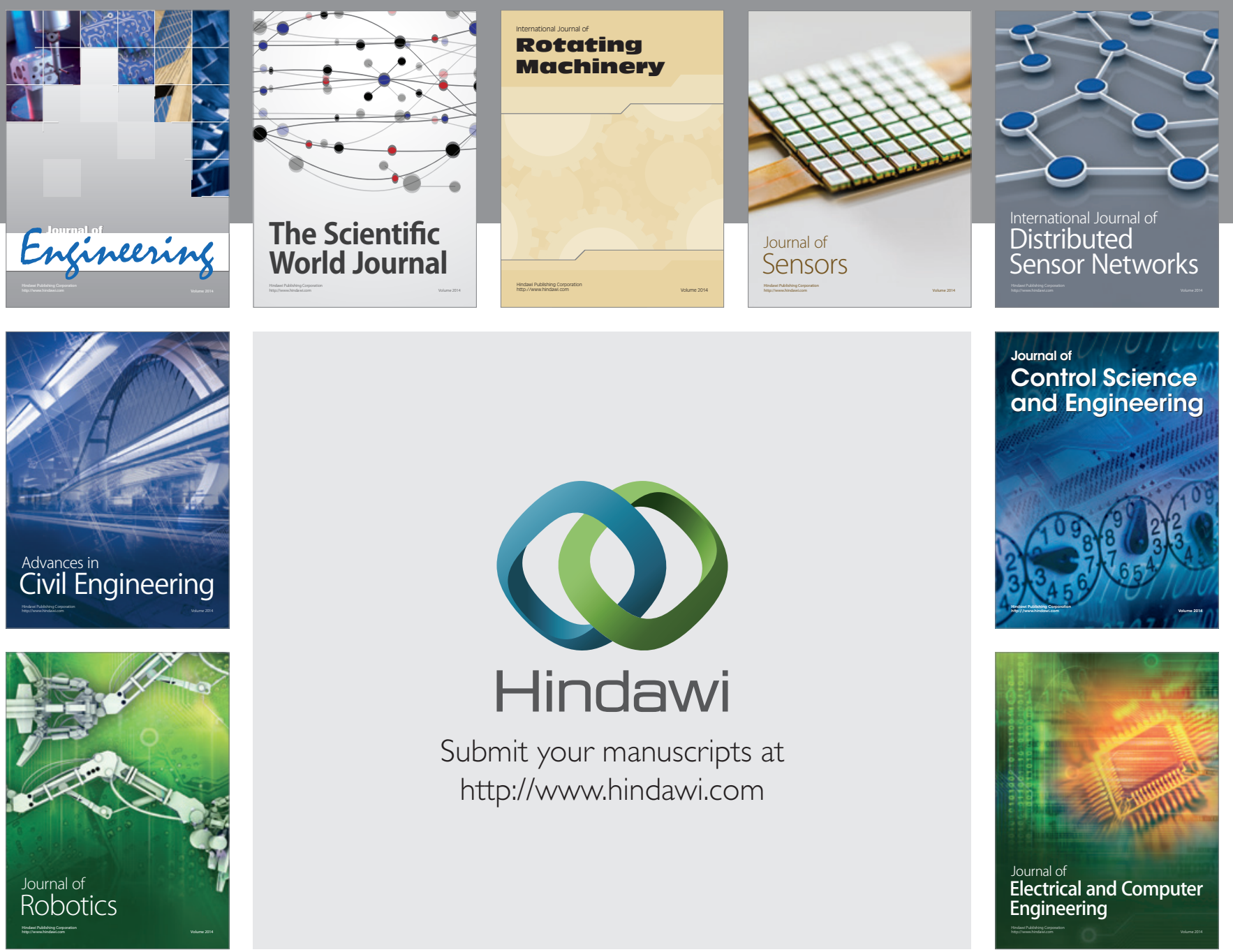

Submit your manuscripts at

http://www.hindawi.com
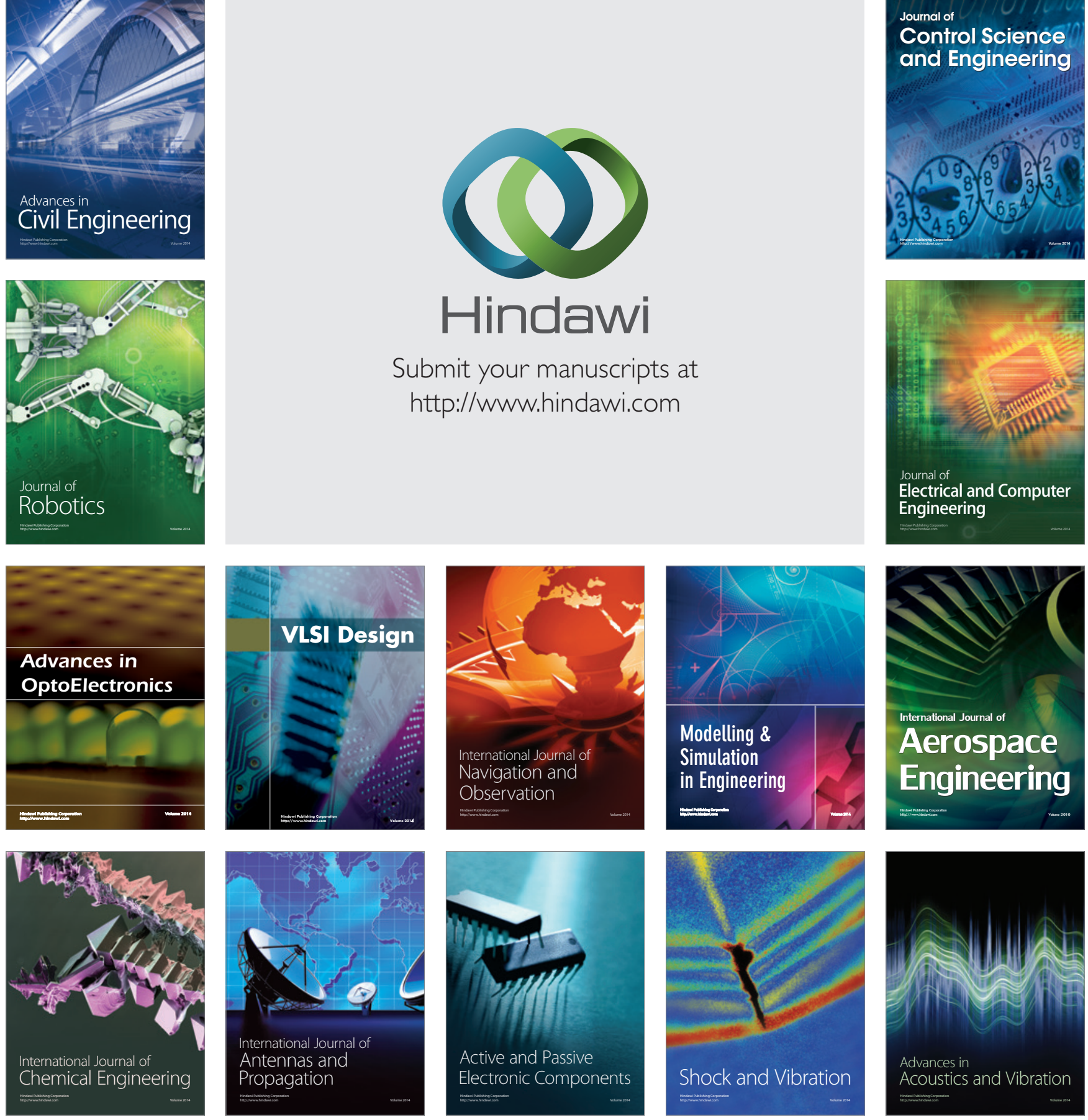